\title{
Monolithic columns with incorporated titanium dioxide nanoparticles for hydrophilic interaction liquid chromatography
}

\author{
N Ganewatta and Z El Rassi* \\ Department of Chemistry, Oklahoma State University, Stillwater, USA.
}

\begin{abstract}
This research article presents the preparation and characterisation of monolithic columns with incorporated titanium dioxide nanoparticles (TDNPs) and their subsequent use in hydrophilic interaction liquid chromatography (HILIC) of small relatively polar solutes. The monolithic support was based on the in situ polymerisation of glyceryl monomethacrylate (GMM) and ethylene glycol dimethacrylate (EDMA) yielding the poly(GMM-co-EDMA) monolith for the incorporation of TDNPs. The poly(GMM-co-EDMA) functioned as a 'true support' for TDNPs' 'stationary phases' as manifested by TDNPs being the major contributor to solute retention, column selectivity and separation efficiencies. In other words, monolithic columns with 'TDNPs stationary phases' were achieved with the understanding that the contribution of the monolith proper to the solute ${ }^{\mathcal{O}}$ retention was at its minimum. The TDNPs were subjected to probe tip highpower sonication at an output power of $10 \mathrm{~W}$ for 20 minutes with the aim of better dispersing the incorporated TDNPs into the monoliths under investigation. In fact, high-power sonication yielded columns with a relatively higher plate count ( $\sim 3$-fold increase) when compared to low-power sonication. Overall, the prepared TDNPs stationary phases proved useful in HILIC of small polar solutes including dimethyl formamide, formamide, thiourea, nucleobases and nucleosides.
\end{abstract}

Keywords: High performance liquid chromatography, hydrophilic interaction liquid chromatography, organic polymer based monolith, titanium dioxide nanoparticles.

\section{INTRODUCTION}

Hydrophilic interaction liquid chromatography (HILIC), is a term that was first coined by Alpert (1990) to a chromatographic technique, which proved a valuable complimentary to reversed phase liquid chromatography for separating polar compounds including small peptides, nucleic acids, carbohydrates, pharmaceutical drugs and small polar-neutral or charged solutes (Alpert, 1990; Jiang et al., 2007). In HILIC, a polar stationary phase and a less polar hydroorganic mobile phase containing more than $50 \%$ organic solvent [e.g., acetonitrile ACN)] are used (Staňková et al., 2013). It is well accepted that an aqueous layer is adsorbed to the surface of the polar stationary phase so that polar analytes will undergo partitioning between the semi-immobilised aqueous layer and the mobile phase while going down the column. Polar solutes have higher affinities towards the semi-immobilised aqueous layer than the organicrich mobile phase, which lead to increased retention in a HILIC separation. In this retention process, hydrogen bonding, dipole-dipole interactions and electrostatic interactions contribute to solute retention, thus resulting in a multimodal HILIC separation mechanism (Alpert, 1990; Buszewski \& Noga, 2012; Gama et al., 2012).

Due to their unique properties such as high permeability, low resistance to mass transfer, ease of preparation with readily available monomers, ability to be confined in columns of all sizes and high stability even at extreme $\mathrm{pH}$ conditions, polymer monolithic stationary phases are preferred over particle packed columns and are becoming more and more popular in HPLC separations (Svec, 2004; Guiochon, 2007).

*Corresponding author (ziad.el_rassi@okstate.edu; (10) https://orcid.org/0000-0001-8018-6244) 
Many attempts have been made for the preparation of organic polymer based monolithic stationary phases for separating polar molecules by the HILIC mode, and acrylamide based (Guryča et al., 2007) and methacrylate based monoliths (Jiang et al., 2007) have been used for that purpose. HILIC monolithic columns have been limited to these two types of monoliths mainly because of the lack of commercially available polar monomers and the low solubility of the available monomers in commonly used porogens (Jiang et al., 2007; 2011).

Although organic polymer monoliths have various advantages over packed columns, the separation of small solutes on these organic polymer monoliths is challenging due to the absence of the required mesoporous structure for small molecule separations (Nischang et al., 2011). Therefore, it is necessary to modify the native polymer monoliths to circumvent the abovementioned drawback of the organic polymer monolithic stationary phases. One such promising approach is the incorporation of nanostructured materials into the monolith thereby combining the unique properties of the nanomaterials such as large surface-to-volume ratio and various surface adsorption properties, with the outstanding characteristics of the organic polymer based monoliths (Xu et al., 2010; Tong et al., 2013).

In the field of chromatography, nanoparticles of carbon, metal, metal oxide and polymer are currently used in the preparation of stationary phases with incorporated nano-entities (Nesterenko et al., 2013). In particular, metal oxide modified stationary phases yield enhanced separation selectivities due to the amphoteric properties and superior chemical affinities exhibited by the metal oxides (Tang et al., 2014). Among them, titanium dioxide offers a wide range of $\mathrm{pH}$ stability compared to silica monoliths, and also possesses biocompatibility, thermal and mechanical stability and the ability of binding phosphorylated peptides and proteins (Cernigoj et al., 2016). Furthermore, the nanoparticles of titanium dioxide have been successfully used in capillary electrophoresis (CE), capillary electrochromatography (CEC) and HPLC.

Zhou et al. (2008) were the first to use titanium dioxide nanoparticles (TDNPs) as run buffer additives in CE. They separated eight $\beta$-adrenergic drugs and observed an increased electroosmotic flow due to the addition of TDNPs to the running electrolyte resulting in a shorter analysis time. Furthermore, Hsieh and coworkers prepared a TDNP-coated column for open tubular CEC separation of angiotensin type oligopeptides with an average separation efficiency of 31,000 plates $/ \mathrm{m}$ (Hsieh et al., 2005).
Open tubular columns coated with nanoparticles seem to be a promising separation approach for proteins mainly due to their large surface-to-volume ratio (Zhang et al., 2016). In fact, a TDNP-coated open tubular capillary column allowed the separation of the main peak of ovalbumin in egg white with 10,000 plates/m (Hsieh et al., 2006). Also, a film of TDNPs was deposited onto the surface of a pre-modified fused silica capillary column with polydopamine, by a liquid phase deposition process, and the resulting column was successfully used in the separation of two variants of $\beta$-lactoglobulin and eight glycoisoforms of ovalbumin (Zhang et al., 2016).

Nanoparticles of rutile, which is a polymorph of titanium dioxide were recently immobilised onto an organic monolithic support for the first time, which was functionalised with aliphatic diol groups. The columns thus obtained were used for the enrichment of phosphopeptides (Cernigoj et al., 2016). However, to the best of our knowledge, there is no information reported on the application of organic polymer based monolithic stationary phases with incorporated nanoparticles of titanium dioxide in HILIC separations. Therefore, the objective of this study was to prepare monolithic HPLC columns with incorporated TDNPs by combining the unique properties of organic polymer based monoliths with those of TDNPs. In this regard, commercially available AEROXIDE ${ }^{\circledR} \mathrm{TiO}_{2} \mathrm{P}$ 90 TDNPs were physically entrapped into neutral, polar poly (glyceryl monomethacrylate-co-ethylene glycol dimethacrylate) which is referred to as poly (GMM-coEDMA) monolithic support, and this research article describes the preparation, chromatographic evaluation and the optimum separation conditions of the prepared monolithic HPLC columns.

Evonik's titanium dioxide nanoparticles with the trade name AEROXIDE ${ }^{\circledR} \mathrm{TiO}_{2} \mathrm{P} 90$ are produced by utilising $\mathrm{TiCl}_{4}$ as the raw material.

$$
\mathrm{TiCl}_{4}(\mathrm{~g})+2 \mathrm{H}_{2}(\mathrm{~g})+\mathrm{O}_{2}(\mathrm{~g}) \rightarrow \mathrm{TiO}_{2}(\mathrm{~s})+4 \mathrm{HCl}(\mathrm{g})
$$

In this process, highly pure liquid $\mathrm{TiCl}_{4}$ is vaporised, followed by mixing it with air and $\mathrm{H}_{2}(\mathrm{~g})$. These gases are then immediately reacted at temperatures between 1000 $-2400{ }^{\circ} \mathrm{C}$ in a burner leading to the formation of fumed TDNPs. The TDNPs used in this study have mixed anatase of $80-90 \%$ and rutile crystal structures. They are highly dispersed, non-porous, hydrophilic in nature and have an average primary particle size of $\sim 14 \mathrm{~nm}$ and a specific surface area of $90 \pm 20 \mathrm{~m}^{2} / \mathrm{g}\left(\right.$ AEROXIDE $^{\circledR}$, AERODISP $^{\circledR}$ and AEROPERL ${ }^{\circledR}$ Titanium Dioxide as Photocatalyst-Technical Information 1243). 


\section{METHODOLOGY}

\section{Instruments}

The HPLC system consisted of an ISCO pump Model 2350, a gradient programmer Model 2360, a $\mathrm{V}^{4 \mathbb{B}}$ variable wavelength absorbance detector (ISCO Inc., Lincoln, NE, USA), and a Rheodyne injector Model 7010 from IDEX Health and Science LLC (Rohnert Park, CA, USA) equipped with a $20 \mu \mathrm{L}$ loop. Chromatograms were recorded with a Chromatopac CR601 integrator from Shimadzu (Kyoto, Japan). A constant pressure pump from Shandon Southern Products Ltd. (Cheshire, UK) was used in conditioning the monolithic columns at high pressures. The in situ polymerisation of monolithic HPLC columns was carried out in a water bath equipped with an immersion circulator Model Isotemp 2100 from Fisher Scientific (Waltham, MA, USA). After the polymerisation, the porogens and the unreacted materials were washed off using a Waters pump Model 590 from Waters division of Millipore (Milford, MA, USA). The ultrasonic cleaner model 1510R-MTH from Branson Ultrasonic Cooperation (Danbury, CT, USA) was used for low power sonication and the model F50 Sonic Dismembrator from Fisher Scientific (Waltham, MA, USA) was used for high power sonication.

\section{Reagents and materials}

Glyceryl monomethacrylate (GMM) was purchased from Monomer-Polymer and Dajac Labs (Trevose, PA, USA). Ethylene glycol dimethacrylate (EDMA), cyclohexanol, 1-dodecanol, thiourea, uracil, adenine, cytosine, uridine, adenosine and cytidine were purchased from Sigma Chemical Co. (St. Louis, MO, USA). 2,2'-Azobis(isobutyronitrile) (AIBN) was obtained from Aldrich Chemical Co. (Milwaukee, WI, USA) and thymine was from Nutritional Biochemical Cooperation (Cleveland, OH, USA). N,N-Dimethylformamide (DMF) and formamide were obtained from EM Science (Gibbstown, NJ, USA). ACS grade acetonitrile (ACN) and isopropanol (IPA) were purchased from PharmcoAAPER (Brookfield, CT, USA). TDNPs, namely, $\begin{array}{llll}\text {AEROXIDE}^{\circledR} & \mathrm{TiO}_{2} & \mathrm{P} 90 & \text { were gifted from Evonik }\end{array}$ Corporation (Parsippany, NJ, USA).

\section{Preparation of TDNPs-incorporated poly(GMM-co- EDMA) monolithic HPLC columns}

The method for the preparation of monolithic HPLC columns with entrapped TDNPs was similar to the procedure described by Mayadunne and El Rassi (2014) in developing poly (GMM-co-EDMA) monolithic stationary phases with incorporated multi-walled carbon nanotubes. However, weight percentages of the constituents of the polymerisation mixture were altered slightly in order to accommodate fumed TDNPs without significantly disrupting the monolithic structure. The polymerisation solutions used in this study have a final weight of $3 \mathrm{~g}$. The GMM monomer along with the EDMA crosslinker, the porogen (cyclohexanol and 1-dodecanol), and AIBN were mixed according to the following wt \%, respectively: $17.95,11.96,34.90,34.90$ and 0.30 . To this mixture, various amounts of TDNPs were added in order to examine the effects of the amount of incorporated TDNPs on solute retention, selectivity and separation efficiencies. In this regard, TDNPs were added to the binary porogenic mixture comprised of cyclohexanol and 1-dodecanol and the resulting mixture was highpower sonicated using a sonication probe continuously for $20 \mathrm{~min}$ at an output power of $10 \mathrm{~W}$ while keeping the solution vial in an ice bath to prevent evaporation of the solution. Next, GMM and EDMA followed by AIBN were added into the polymerisation solution, which was then vortexed for $2 \mathrm{~min}$ and degassed in a low-power sonication bath for another $20 \mathrm{~min}$. The polymerisation mixture appeared homogenous and free of air bubbles. Thereafter, this final polymer solution was introduced into a two-section $15-\mathrm{cm}$ long stainless steel column consisting of a $10 \mathrm{~cm} \times 4.6 \mathrm{~mm}$ i.d. section connected with a $1 / 4$ inch union to a $5 \mathrm{~cm} \times 4.6 \mathrm{~mm}$ i.d. section. This extra $5-\mathrm{cm}$ long column was added to ensure the complete formation of the $10-\mathrm{cm}$ long monolithic column without any voids or contractions in it. Next, the column end fittings were sealed with end plugs and the polymerisation was allowed to proceed at $60^{\circ} \mathrm{C}$ for $15 \mathrm{~h}$ in a water bath according to the following Scheme.

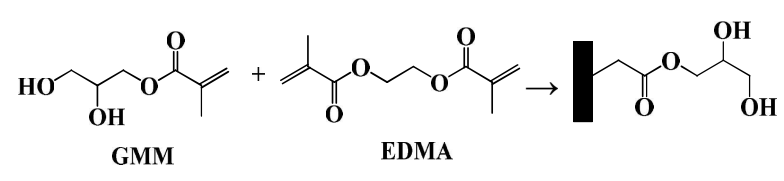

After completion of the polymerisation, the end plugs were taken off and the column was washed with ACN to remove the porogens and the unreacted monomers using a Waters HPLC pump. Then, the column was equilibrated with IPA for $3 \mathrm{~h}$ using the same HPLC pump. The monolithic column thus obtained was connected to a constant pressure pump and a pressure of $6000-7000$ psi was applied for 20 min using IPA as the solvent. The application of high pressure was to make sure that possible voids and contractions were removed from the monolithic structure in the $10-\mathrm{cm}$ long column. Thereafter, the top 5-cm column was separated from the 10-cm long analytical column. This analytical column was equilibrated with ACN prior to the HPLC analyses. 
A blank 10-cm long monolithic column with no TDNPs was also prepared in the same way.

In order to study the effect of the sonication mode on the column retentive properties and the separation efficiencies, another column was prepared by following the composition of the column designated as $\mathrm{TiO}_{2}-50 \mathrm{mg}$ in Table 1. In the preparation of this column, the TDNPs were first added to the porogenic mixture and the polymerisation solution was only low-power sonicated for 20 min after adding GMM, EDMA and AIBN. Finally, the polymerisation solution thus obtained was introduced into the $15-\mathrm{cm}$ long stainless steel column and treated in the same way as just described in the preceding paragraph.

\section{RESULTS AND DISCUSSION}

\section{Poly (GMM-co-EDMA) monolithic support with} physically entrapped fumed TDNPs

In this study, fumed TDNPs were physically incorporated into a relatively polar poly (GMM-co-EDMA) monolithic support shown in the Scheme above. In order to find the optimum amount of TDNPs that can be physically entrapped into the monolithic HPLC columns, a series of compositions was tested by simply varying the amount of TDNPs that was incorporated into the polymerisation solution.

Furthermore, when the prepared monolithic columns were compressed into the $10-\mathrm{cm}$ long column using the constant high pressure pump, different compressibilities, which were strongly dependent on the amount of the TDNPs incorporated into the polymerisation solution, were observed. In fact, the monolith with a low amount of TDNPs compressed more than the one with a high amount of entrapped TDNPs. Due to the differences in compressibilities of the prepared monoliths, the actual amounts of the TDNPs incorporated into the 10-cm long analytical column were not proportional to the amount of TDNPs added into the polymerisation solution. Thus, the actual masses of the TDNPs present in the $10-\mathrm{cm}$ long separation columns were estimated by considering the final length of the resulting monolithic columns after the compression step as summarised in Table 1.

The backpressure measurement data obtained on the monolithic columns with physically entrapped fumed TDNPs indicated that the formation of the monolithic structure was strongly related to the fumed TDNPs content in the polymerisation mixture. For example, the incorporation of $3.8 \mathrm{mg}$ of TDNPs/cm of the monolithic column resulted in an increased backpressure of 1.6 fold with respect to the blank column at a flow rate of $1.8 \mathrm{~mL} / \mathrm{min}$ of pure $\mathrm{ACN}$ as the mobile phase. A linear relationship (coefficient of determination, $\mathrm{R}^{2}>0.99$ ) between the mobile phase flow rate and the resulting back pressure obtained on the column was observed in the flow rate range of 0.6 to $1.9 \mathrm{~mL} / \mathrm{min}$ with a mobile phase of pure ACN. This is a clear indication of the good mechanical stability of the monolithic columns with incorporated TDNPs.

Table 1: Estimation of the actual amount of TDNPs in the analytical columns. Volume of $10+5 \mathrm{~cm}$ column, $2.49 \mathrm{~mL}$; volume of $10 \mathrm{~cm}$ column, $1.66 \mathrm{~mL}$

\begin{tabular}{|c|c|c|c|c|c|c|}
\hline $\begin{array}{l}\text { Weight of } \\
\text { TDNPs } \\
\text { (mg) }\end{array}$ & $\begin{array}{l}\text { Weight of TDNPs in } \\
(10+5) \mathrm{cm} \text { column } \\
(\mathrm{mg})\end{array}$ & $\begin{array}{l}\text { Monolithic length } \\
\text { in } 5 \mathrm{~cm} \text { column } \\
\text { after compression } \\
\qquad(\mathrm{cm})\end{array}$ & $\begin{array}{c}\text { Total length of } \\
\text { monolithic }(10+5) \\
\text { cm column after } \\
\text { compression }(\mathrm{cm})\end{array}$ & $\begin{array}{l}\text { Volume of total } \\
\text { length of compressed } \\
\text { monolithic column } \\
(\mathrm{mL})\end{array}$ & $\begin{array}{l}\text { Actual mass of } \\
\text { TDNPs in } 10 \mathrm{~cm} \\
\text { column }(\mathrm{mg})\end{array}$ & $\begin{array}{l}\text { Mass of TDNPs } \\
\text { per unit column } \\
\text { length }(\mathrm{mg} / \mathrm{cm})\end{array}$ \\
\hline 25.0 & 20.78 & 0.5 & 10.5 & 1.75 & 19.79 & 2.0 \\
\hline 37.5 & 31.13 & 0.8 & 10.8 & 1.80 & 28.77 & 2.9 \\
\hline 50.0 & 41.56 & 1.0 & 11.0 & 1.83 & 37.79 & 3.8 \\
\hline 60.0 & 49.88 & 2.0 & 12.0 & 2.00 & 41.56 & 4.2 \\
\hline 75.0 & 62.35 & 3.0 & 13.0 & 2.16 & 47.96 & 4.8 \\
\hline
\end{tabular}

Optimum amount of TDNPs physically incorporated into the poly(GMM-co-EDMA) monolithic support

In order to find out the optimum amount of TDNPs that can be incorporated into the desired monolithic support, the separation efficiencies of the columns with varied amounts of incorporated TDNPs were evaluated using DMF, formamide and thiourea as model solute probes. The calculated separation efficiencies of these neutral polar solutes are plotted in Figure 1. The monolithic 
HPLC column with incorporated fumed TDNPs at $3.8 \mathrm{mg} / \mathrm{cm}$ of column length yielded the maximum average plate count $/ \mathrm{m}$ for the tested neutral polar solutes. Therefore, the incorporation of $50 \mathrm{mg}$ of fumed TDNPs into the polymerisation solution, which produces $3.8 \mathrm{mg}$ of TDNPs per cm of column length was selected as the optimum amount of the TDNPs that can be physically incorporated into the poly(GMM-co-EDMA) monolithic support and this column was used in the rest of this investigation. The control/blank column yielded the lowest separation efficiency of the test solutes confirming the ability of obtaining enhanced separation efficiencies due to the incorporation of TDNPs into the monolithic columns. In addition, variation of the retention factors, $\mathrm{k}$, of the tested polar solutes upon increasing the content of the TDNPs per unit column length (i.e., mg of TDNPs/cm column length) is shown in Figure 2. As can be seen in Figure 2, the $\mathrm{k}$ values for the tested polar solutes fluctuated as a function of the amount of TDNPs added. However, for the 3 solutes, the $\mathrm{k}$ values peaked slightly at $3.8 \mathrm{mg}$ of TDNPs $/ \mathrm{cm}$. This observation further justifies the selection of $50 \mathrm{mg}$ as the optimum content of TDNPs that can be incorporated into the polymerisation solution yielding $3.8 \mathrm{mg}$ of TDNPs/cm of the monolithic column.

\section{Chromatographic evaluations of monolithic HPLC column with incorporated fumed TDNPs}

The retention characteristics of the monolithic HPLC columns with incorporated TDNPs were evaluated with some neutral polar solutes (e.g., thiourea, DMF and formamide), nucleobases and nucleosides as model solute probes and the analyses were performed on the monolithic column which contained $3.8 \mathrm{mg} / \mathrm{cm}$ of TDNPs. The separation efficiencies of these tested analytes were compared with the separation efficiencies obtained for the blank column with no incorporated fumed TDNPs.

\section{Small polar neutral solutes.}

Mobile phases containing relatively high organic content are used in HILIC analyses. Therefore, a mobile phase comprising $95 \% \mathrm{ACN}(\mathrm{v} / \mathrm{v})$ and $5 \%$ water (v/v) was used for the analysis of toluene ( $t_{0}$ marker), DMF, formamide and thiourea as the test compounds to evaluate the HILIC retention behaviour of the prepared monolithic columns without (Figure 3A) and with incorporated fumed TDNPs (Figure 3B). The most non-polar solute toluene eluted first at the deadtime of the column (i.e., deadtime marker) and the remaining three solutes were eluted in the order of increasing polarity, showing a typical HILIC

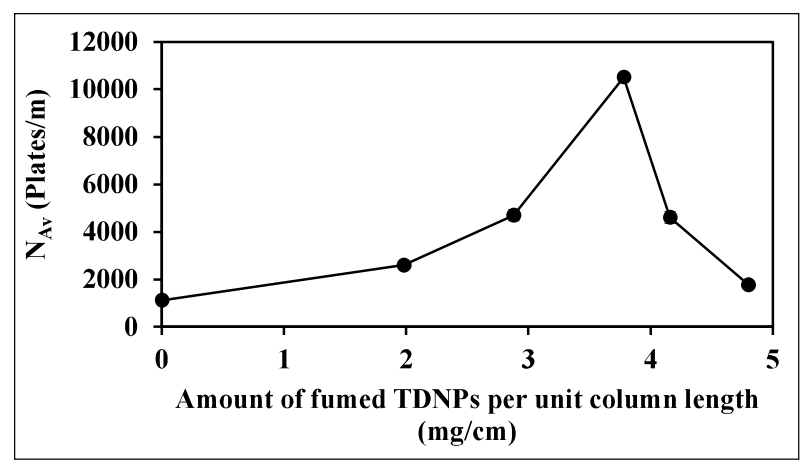

Figure 1: Average plate count $/ \mathrm{m}$ vs. amount of fumed TDNPs per unit column length obtained on different monolithic columns with varied amounts of incorporated fumed TDNPs

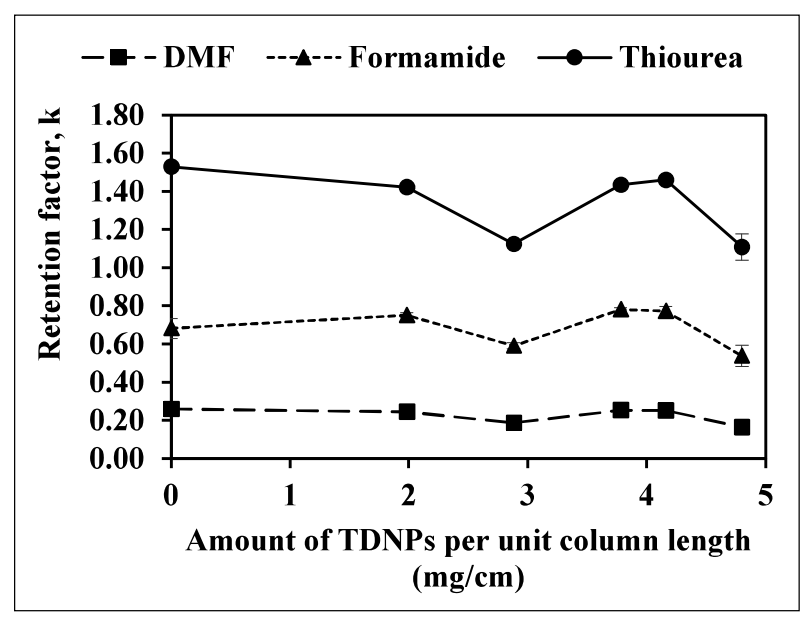

Figure 2: Dependence of the solute retention factor, k, of some neutral polar solutes on the amount of incorporated TDNPs per unit column length. Conditions: column, $10 \mathrm{~cm} \times 4.6 \mathrm{~mm}$ i.d.; mobile phase, $95 \% \mathrm{ACN}(\mathrm{v} / \mathrm{v}), 5 \%$ water (v/v); flow rate, $1 \mathrm{~mL} / \mathrm{min}$; injection volume, $20 \mu \mathrm{L}$; temperature, room temperature.

behaviour where the least polar DMF eluted first. The most polar solute thiourea, which is commonly used as the unretained marker in RPC analyses eluted last. It should be noted that the values of the selectivity factor, $\alpha$, were noticeably as high as 3.1 and 1.8 for the solute pairs 2/1 (i.e., formamide/DMF) and $3 / 2$ (i.e., thiourea/ formamide), respectively, indicating a good separation for these solutes, although the peaks are relatively broad as can be seen in Figure 3B. In addition, the variation of the $\mathrm{k}$ values of these tested analytes upon increasing the TDNPs content of the column, was also studied (Figure 2). 


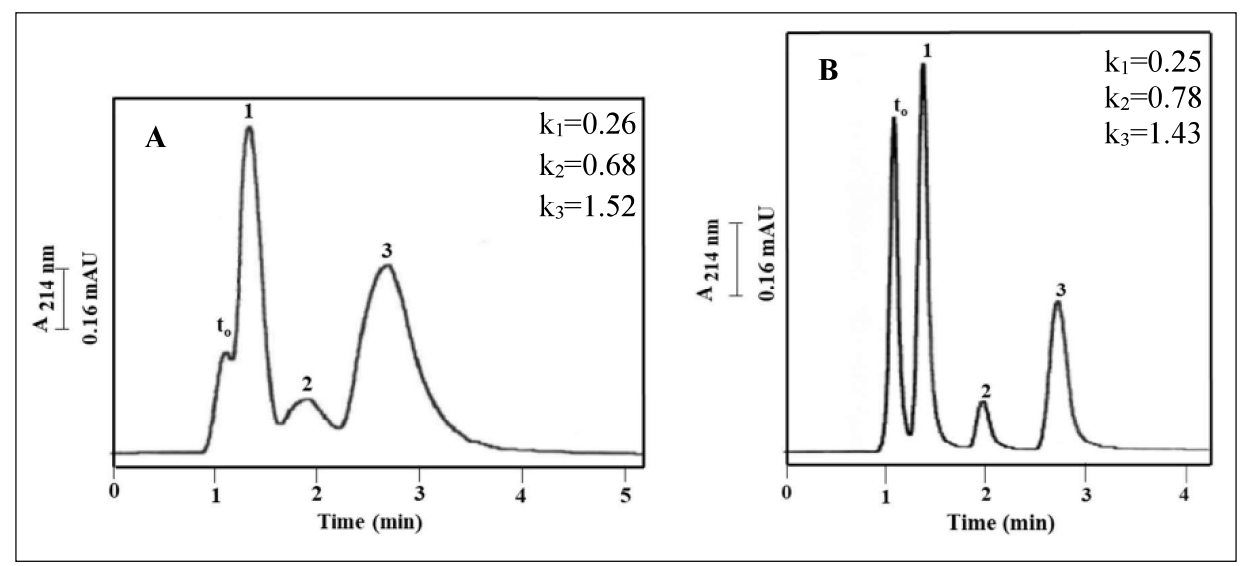

Figure 3: Chromatograms of small neutral polar solute probes obtained on the monolithic columns without (A) and with (B) incorporated fumed TDNPs at $3.8 \mathrm{mg}$ of fumed TDNPs/ $\mathrm{cm}$. Conditions: mobile phase, $95 \% \mathrm{ACN}(\mathrm{v} / \mathrm{v}), 5 \%$ water (v/v); flow rate, $1 \mathrm{~mL} / \mathrm{min}$; injection volume, $20 \mu \mathrm{L}$; temperature, room temperature. Solutes: $\mathrm{t}_{0}$, toluene; 1 , DMF; 2 , formamide; 3 , thiourea

Moreover, as can be seen in Figure 1, a separation efficiency of 10,600 plates $/ \mathrm{m}$ was obtained for the column under investigation in separating the solute probes under investigation. Under similar testing conditions, the blank column yielded only 1,100 plates $/ \mathrm{m}$ for the separation of the solute probes. This result further confirms the enhancement of the separation efficiencies of the monolithic columns by incorporating TDNPs.

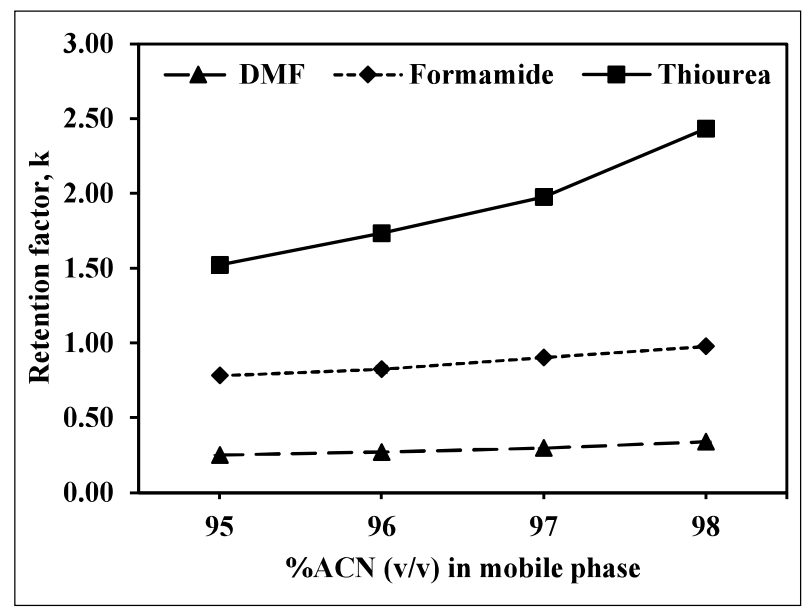

Figure 4: Plot of the k values vs. $\% \mathrm{ACN}(\mathrm{v} / \mathrm{v})$ in the mobile phase obtained on the column with $3.8 \mathrm{mg}$ of TDNPs per $\mathrm{cm}$ column length. Conditions: column, $10 \mathrm{~cm} \times 4.6 \mathrm{~mm}$ i.d.; flow rate, $1 \mathrm{~mL} / \mathrm{min}$; detection, $\mathrm{UV}$ at $214 \mathrm{~nm}$; injection volume, $20 \mu \mathrm{L}$; column temperature, room temperature; mobile phase, $\mathrm{ACN}$ with varying percentages of water
In HILIC, the eluting strength of the mobile phase decreases with increasing organic content in the mobile phase. In order to evaluate the effect of the organic content (i.e., ACN content) of the mobile phase on solute retention, the $\mathrm{k}$ values of DMF, formamide and thiourea were calculated at various percentages of $\mathrm{ACN}$ in the mobile phase, and the results are shown in Figure 4. The retention factors of all these solutes increased with increasing the organic content in the mobile phase showing the typical HILIC behaviour.

\section{Nucleobases}

To further characterise the monolith with physically entrapped TDNPs, a mixture of 4 nucleobases consisting of uracil, thymine, adenine and cytosine was chromatographed on the column under investigation yielding a separation efficiency of 3,400 plates $/ \mathrm{m}$. These weakly basic solutes were eluted following the order of thymine, uracil, adenine and cytosine when using the mobile phase composed of $95 \%(\mathrm{v} / \mathrm{v}) \mathrm{ACN}$ and $5 \%(\mathrm{v} / \mathrm{v})$ of $10 \mathrm{mM} \mathrm{CH}_{3} \mathrm{COONH}_{4}, \mathrm{pH} 7.0$ (Figure $5 \mathrm{~B})$. For comparison, the chromatogram obtained on the blank monolithic column yielded only 1,350 plates $/ \mathrm{m}$ (Figure 5A). The selectivity factors were remarkably high at 1.2, 4.4 and 1.7 for the solute pairs $2 / 1,3 / 2$ and $4 / 3$, respectively, and as a result the peaks were well separated as shown in Figure 5B. Moreover, the k values of the nucleobases obtained on the monolithic columns prepared with varied amounts of incorporated TDNPs were also evaluated. The resulted $\mathrm{k}$ values fluctuated as a function of the amount of incorporated TDNPs peaking up slightly at around $3.8-4.2 \mathrm{mg}$ TDNPs $/ \mathrm{cm}$. 


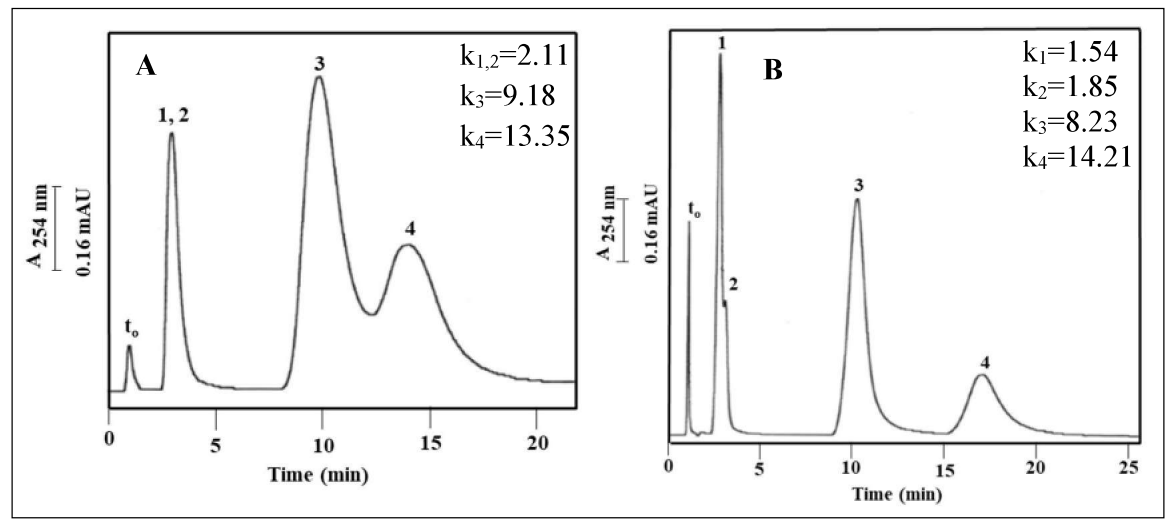

Figure 5: Chromatograms of nucleobases obtained on monolithic columns without (A) and with (B) incorporated fumed TDNPs at $3.8 \mathrm{mg}$ of fumed TDNPs $/ \mathrm{cm}$. Conditions: mobile phase, $95 \% \mathrm{ACN}(\mathrm{v} / \mathrm{v}), 5 \%$ (v/v) of $10 \mathrm{mM} \mathrm{CH}_{3} \mathrm{COONH}_{4}$, $\mathrm{pH} 7.0$; flow rate, $1 \mathrm{~mL} / \mathrm{min}$; injection volume, $20 \mu \mathrm{L}$; temperature, room temperature. Solutes; $\mathrm{t}_{0}$, toluene; 1 , thymine; 2 , uracil; 3 , adenine; 4, cytosine

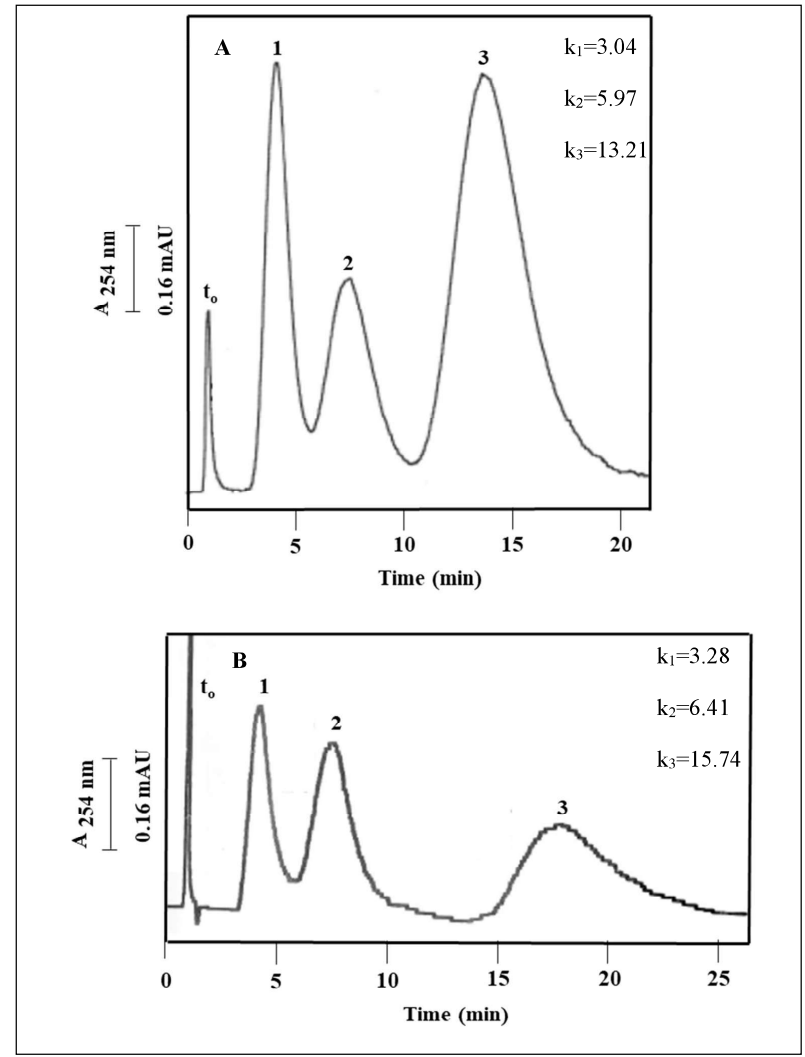

Figure 6: Chromatograms of nucleosides obtained on monolithic column without (A) and with (B) incorporated fumed TDNPs at $3.8 \mathrm{mg}$ of fumed TDNPs/cm. Solutes; $\mathrm{t}_{0}$, toluene; 1, uridine; 2, adenosine; 3 , cytidine. Other conditions are the same as in Figure 5.

\section{Nucleosides}

Three nucleosides, namely, uridine, adenosine and cytidine were chromatographed on the same column (i.e., with $3.8 \mathrm{mg}$ of TDNPs/cm of column) using the same mobile phase conditions as those for the separation of nucleobases. Nucleosides are considered as very polar solutes consisting of a nitrogenous base covalently attached to either a ribose or deoxyribose sugar. Adenosine $\left(\mathrm{pK}_{\mathrm{a}}=3.64\right)$ and cytidine $\left(\mathrm{pK}_{\mathrm{a}}=4.22\right)$ are weakly basic in nature due to the proton gaining power of the nitrogen atoms in their corresponding nucleobases, whereas uridine $\left(\mathrm{pK}_{\mathrm{a}}=9.3\right)$ stays neutral over a wide range of $\mathrm{pH}$ and may release a proton at very alkaline $\mathrm{pH}$. Therefore, under the used $\mathrm{pH}$ conditions of the aqueous portion of the mobile phase (i.e., $\mathrm{pH} 7$ ), adenosine, cytidine and uridine are neutral in charge. The elution order of these nucleosides was uridine, adenosine and cytidine under the tested isocratic conditions.

Figure 6 displays the chromatograms of these nucleosides obtained on the monolithic HPLC column with incorporated TDNPs (Figure 6B) and its blank column (Figure 6A). These tested nucleosides were separated on the monolithic column with entrapped TDNPs yielding a plate count of 1,200 plates/m while the blank monolithic column, which was devoid of TDNPs yielded only 700 plates $/ \mathrm{m}$ for the same nucleosides. The tested nucleosides were separated on the TDNPincorporated monolithic column with $\alpha$ values of 1.7 and 2.7 for the solute pairs $2 / 1$ and $3 / 2$, respectively. 
Furthermore, higher $\mathrm{k}$ values were expected for the nucleosides compared to their nucleobase counterparts due to the hydrophilicity arising from the covalently attached sugar moiety in nucleosides. For instance, the $\mathrm{k}$ values observed for uracil, uridine, cytosine and cytidine were $1.85,3.28,14.21$ and 15.74 , respectively. As anticipated, uridine and cytidine gave higher $\mathrm{k}$ values than their nucleobase counterparts uracil and cytosine. However, adenosine exhibited lower k value compared to adenine. This deviation from the expected results may be due to the combined effect of various types of interactions including hydrogen bonding, electrostatic, ion-dipole and dipole-dipole interactions which are involved in the separation mechanism of HILIC stationary phases that makes the prediction of the elution order difficult. The variation of the $\mathrm{k}$ values of the nucleosides obtained on the monolithic columns upon increasing the TDNPs content is shown in Figure 7. In all the chromatographic analyses, the tested solutes were well resolved on the column with incorporated TDNPs when compared to the blank column with no TDNPs in it. Therefore, the incorporated TDNPs have played a significant role in separating the model analytes with enhanced resolution under the tested HILIC conditions.

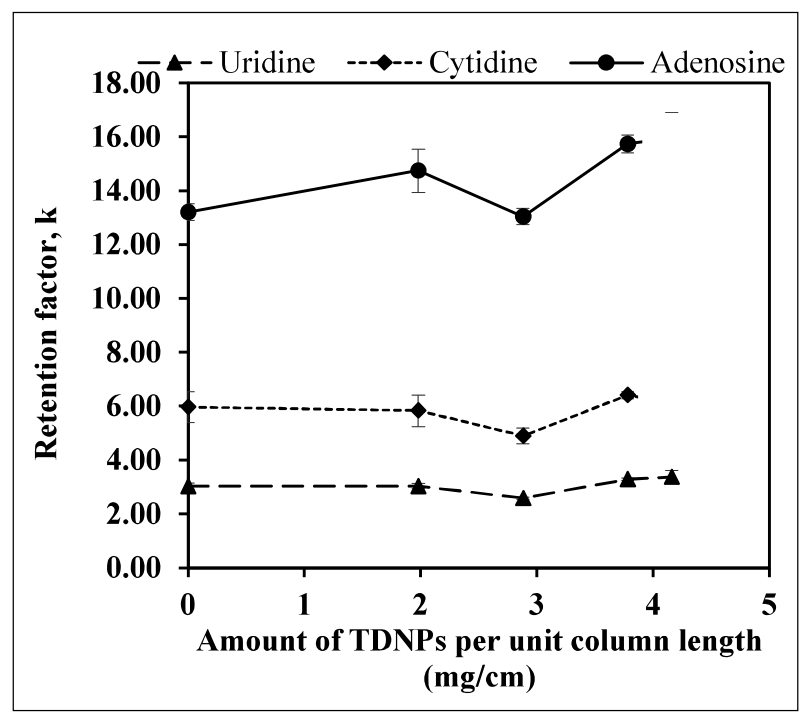

Figure 7: Dependence of the solute retention factor, $k$, of some nucleosides on the amount of incorporated TDNPs per unit column length. Other conditions are the same as in Figure 5.

\section{Effect of sonication on retention characteristics}

Nanoparticles tend to aggregate with each other easily due to the van der Waals interactions arising from the large specific surface of their sub-micron particles. It is essential to disperse these nanoparticles uniformly in the polymerisation solution in order to obtain homogeneous monolithic structures. In this regard, ultrasonication has been proven as a powerful mechanical tool, which disperses the nanoparticles homogeneously in solution by preventing their agglomerations (Othman et al., 2012; Qi et al., 2012). This dispersing ability of the ultrasonication occurs due to acoustic cavitation, which is responsible for a high-energy input. The nanoparticle de-agglomeration takes place when the energy released from the collapses of the cavitation bubbles is transferred to the suspensions (Suslick, 1990; Zhang et al., 2011).

Previous studies reported the successful dispersion of TDNPs suspensions by ultrasonication (Wieneke et al., 2011; Zhang et al., 2011; Qi et al., 2012). In particular, Qi et al. (2012) have obtained a stable aqueous suspension of TDNPs using ultrasonication for $20 \mathrm{~min}$ at $180 \mathrm{~W}$. Therefore, in this study, the binary porogenic mixture containing TDNPs was high-power sonicated for $20 \mathrm{~min}$ before adding the functional monomer and the crosslinker. Furthermore, the effect of the applied mode of sonication (i.e., low-power bath sonication or high-power probe tip sonication) in the preparation of the polymerisation solution mixture on the retention properties of the resulting column was examined by comparing the $\mathrm{k}$ values obtained on two columns each containing $3.8 \mathrm{mg}$ fumed TDNPs $/ \mathrm{cm}$ of column length. One monolithic HPLC column was prepared by high power sonication of the fumed TDNPs in the binary porogenic mixture for $20 \mathrm{~min}$ followed by low power sonication for another $20 \mathrm{~min}$ after adding the monomer, crosslinker and the initiator, whereas the second column was prepared by applying low power sonication for 20 min to the polymerisation solution containing TDNPs. Both columns were tested using the same set of analytes as in the preceding section and the measured $\mathrm{k}$ values are shown in Table 2. For all the tested analytes, the $\mathrm{k}$ values increased by $\sim 16-42 \%$ due to the application of high power sonication at an output of $10 \mathrm{~W}$ for a short time (i.e., $20 \mathrm{~min}$ ) when preparing the polymerisation solution mixture.

According to a recent study, smaller sized particles have been obtained from direct probe tip sonication than from the 'indirect' bath type sonication when performing the dispersion of AEROXIDE $\mathrm{TiO}_{2}$ P25 TDNPs in solution (Meißner et al., 2014). Therefore, in our case the TDNPs might have been broken into smaller particles yielding high specific surface area, which resulted in enhanced interactions with the analytes leading to increased $\mathrm{k}$ values during the chromatographic analyses. 
Table 2: Comparison of the $\mathrm{k}$ values obtained on the monolithic columns prepared by low-power and high-power sonication

\begin{tabular}{|c|c|c|c|c|}
\hline \multirow[b]{2}{*}{ Solute } & \multicolumn{2}{|c|}{ Retention factor, $\mathrm{k}$} & \multirow[b]{2}{*}{$\eta=\mathrm{k}_{\text {High power }} / \mathrm{k}_{\text {Low power }}$} & \multirow{2}{*}{$\begin{array}{l}\% \text { Increase of } \mathrm{k} \text { due to the } \\
\text { high power sonication }\end{array}$} \\
\hline & $\begin{array}{l}\text { High power sonication } \\
\qquad\left(\mathrm{k}_{\text {High power }}\right)\end{array}$ & $\begin{array}{c}\text { Low power sonication } \\
\left(\mathrm{k}_{\text {Low power }}\right)\end{array}$ & & \\
\hline DMF & $0.25( \pm 0.00)$ & $0.19( \pm 0.01)$ & 1.32 & 31.6 \\
\hline Formamide & $0.78( \pm 0.01)$ & $0.62( \pm 0.02)$ & 1.26 & 25.8 \\
\hline Thiourea & $1.43( \pm 0.01)$ & $1.19( \pm 0.04)$ & 1.20 & 20.2 \\
\hline Thymine & $1.54( \pm 0.00)$ & $1.22( \pm 0.05)$ & 1.26 & 26.2 \\
\hline Uracil & $1.85( \pm 0.01)$ & $1.43( \pm 0.04)$ & 1.29 & 29.4 \\
\hline Adenine & $8.23( \pm 0.12)$ & $6.61( \pm 0.25)$ & 1.25 & 24.5 \\
\hline Cytosine & $14.21( \pm 0.20)$ & $12.29( \pm 0.90)$ & 1.16 & 15.6 \\
\hline Uridine & $3.28( \pm 0.05)$ & $2.38( \pm 0.01)$ & 1.38 & 37.8 \\
\hline Adenosine & $6.41( \pm 0.13)$ & $4.52( \pm 0.22)$ & 1.42 & 41.8 \\
\hline Cytidine & $15.74( \pm 0.34)$ & $12.27( \pm 0.39)$ & 1.28 & 28.3 \\
\hline
\end{tabular}

However, the column prepared by applying low-power sonication on the polymerisation mixture yielded only 8,300 plates $/ \mathrm{m}$ for the same set of solutes under the same elution conditions. Therefore, due to the application of high-power sonication, the tested set of small polar neutral solutes were separated with $\sim 1.3$ times higher separation efficiency compared to the columns prepared using low-power sonication. In addition, the separation efficiencies of nucleobases were 3,400 plates/m (Figure 5B) and 3,300 plates $/ \mathrm{m}$ for the monolithic columns prepared by high-power sonication and low-power sonication, respectively. Furthermore, nucleosides were separated with an average plate count of 1,200 plates $/ \mathrm{m}$ (Figure 6B) on the monolithic HPLC column, which was prepared by applying high-power sonication. Conversely, a separation efficiency of only 400 plates $/ \mathrm{m}$ was achieved on the column that contained the same amount of TDNPs prepared by applying low-power sonication. Therefore, the separation efficiency of the tested nucleosides was enhanced by three-fold due to the application of highpower sonication.

\section{Column reproducibility}

The reproducibility of analyses from run-to-run, day-to-day and column-to-column were measured on poly(GMM-co-EDMA) monolithic column with incorporated $3.8 \mathrm{mg}$ of TDNPs per $\mathrm{cm}$ column length using high-power sonication. DMF, formamide and thiourea were used as the model solutes. The $\mathrm{k}$ values of these polarsolute probes were measured on the same column from run-to-run $(\mathrm{n}=3)$ and day-to-day $(\mathrm{n}=3)$ and on different columns i.e., from column-to-column $(\mathrm{n}=3)$ using a mobile phase of $95 \%(\mathrm{v} / \mathrm{v}) \mathrm{ACN}$ and $5 \%(\mathrm{v} / \mathrm{v})$ water. The day-to-day and column-to-column reproducibilities were calculated by averaging triplicate measurements of the $\mathrm{k}$ values on each day and on each column. In all the cases, the reproducibility was determined by the $\%$ relative standard deviation ( $\%$ RSD) of the $\mathrm{k}$ values. The \% RSD for the $\mathrm{k}$ values of the aforementioned three solutes were on the average of $0.9 \%, 1.6 \%$ and $3.1 \%$ from run-to-run, day-to-day and column-to-column, respectively, which reflect the stability and the reliability of making monolithic HPLC columns with physically entrapped TDNPs without a significant leakage of the nanoparticles during the analyses.

\section{CONCLUSIONS}

This study presented for the first time the use of TDNPs for the preparation of nano-doped organic polymer monolithic HPLC column for HILIC. The poly(GMMco-EDMA) monolith proved to be an ideal polar support for the physical entrapment of fumed titanium dioxide nanocrystals. The developed columns were successfully used in the separation of relatively small polar molecules including DMF, formamide, thiourea, nucleobases and nucleosides, mainly via hydrophilic interactions. Probe tip high-power sonication has played a significant role in breaking and consequently dispersing the TDNPs homogeneously in the polymerisation solution thus yielding useful monolithic columns. 


\section{REFERENCES}

AEROXIDE $^{\circledR}$, AERODISP $^{\circledR}$ and AEROPERL ${ }^{\circledR}$ Titanium Dioxide as Photocatalyst-Technical Information 1243. Available at https://www.aerosil.com/sites/ lists/RE/DocumentsSI/TI-1243-Titanium-Dioxide-asPhotocatalyst-EN.pdf, Acessed $28^{\text {th }}$ of April 2017.

Alpert A.J. (1990). Hydrophilic-interaction chromatography for the separation of peptides, nucleic acids and other polar compounds. Journal of Chromatography A 499: 177-196. DOI: https://doi.org/10.1007/s00216-011-5308-5

Buszewski B. \& Noga S. (2012). Hydrophilic interaction liquid chromatography (HILIC)-a powerful separation technique. Analytical and Bioanalytical Chemistry 402(1): 231-247. DOI: https://doi.org/10.1007/s00216-011-5308-5

Cernigoj U., Gaspersic J., Fichtenbaum A., Lendero Krajnc N., Vidic J., Mitulovic G. \& Strancar A. (2016). Titanium dioxide nanoparticle coating of polymethacrylate-based chromatographic monoliths for phosphopetides enrichment. Analytica Chimica Acta 942: 146-154.

DOI: https://doi.org/10.1016/j.aca.2016.08.044

Gama M.R., da Costa Silva R.G., Collins C.H. \& Bottoli C.B. (2012). Hydrophilic interaction chromatography. Trends in Analytical Chemistry 37: 48-60.

DOI: https://doi.org/10.1016/j.trac.2012.03.009

Guiochon G. (2007). Monolithic columns in high-performance liquid chromatography. Journal of Chromatography $A$ 1168(1): 101-168. DOI: https://doi.org/10.1016/j.chroma.2007.05.090

Guryča V., Mechref Y., Palm A.K., Michálek J., Pacáková V. \& Novotný M.V. (2007). Porous polyacrylamide monoliths in hydrophilic interaction capillary electrochromatography of oligosaccharides. Journal of Biochemical and Biophysical Methods 70(1): 3-13.

DOI: https://doi.org/10.1016/j.jbbm.2006.11.002

Hsieh Y.L., Chen T.H., Liu C.P. \& Liu C.Y. (2005). Titanium dioxide nanoparticles-coated column for capillary electrochromatographic separation of oligopeptides. Electrophoresis 26(21): 4089-4097.

DOI: https://doi.org/10.1002/elps.200500462

Hsieh Y.L., Chen T.H. \& Liu C.Y. (2006). Capillary electrochromatographic separation of proteins on a column coated with titanium dioxide nanoparticles. Electrophoresis 27(21): 4288-4294.

DOI: https://doi.org/10.1002/elps.200500897

Jiang Z., Smith N.W., Ferguson P.D. \& Taylor M.R. (2007). Hydrophilic interaction chromatography using methacrylate-based monolithic capillary column for the separation of polar analytes. Analytical Chemistry 79(3): 1243-1250.

DOI: https://doi.org/10.1021/ac061871f

Jiang Z., Smith N.W. \& Liu Z. (2011). Preparation and application of hydrophilic monolithic columns. Journal of Chromatography A 1218(17): 2350-2361. DOI: https://doi.org/10.1016/j.chroma.2011.02.024
Mayadunne E. \& El Rassi Z. (2014). Facile preparation of octadecyl monoliths with incorporated carbon nanotubes and neutral monoliths with coated carbon nanotubes stationary phases for HPLC of small and large molecules by hydrophobic and $\pi-\pi$ interactions. Talanta 129: 565-574. DOI: https://doi.org/10.1016/j.talanta.2014.06.032

Meißner T., Oelschlägel K. \& Potthoff A. (2014). Dispersion of nanomaterials used in toxicological studies: a comparison of sonication approaches demonstrated on $\mathrm{TiO}_{2} \mathrm{P} 25$. Journal of Nanoparticle Research 16(2): 2228.

DOI: https://doi.org/10.1007/s11051-013-2228-7

Nesterenko E.P., Nesterenko P.N., Connolly D., He X., Floris P., Duffy E. \& Paull B. (2013). Nano-particle modified stationary phases for high-performance liquid chromatography. Analyst 138(15): 4229-4254.

DOI: https://doi.org/10.1039/c3an00508a

Nischang I., Teasdale I. \& Brüggemann O. (2011). Porous polymer monoliths for small molecule separations: advancements and limitations. Analytical and Bioanaytical Chemistry 400(8): 2289-2304.

DOI: https://doi.org/10.1007/s00216-010-4579-6

Othman S.H., Rashid S.A., Ghazi T.I.M. \& Abdullah N. (2012). Dispersion and stabilization of photocatalytic $\mathrm{TiO}_{2}$ nanoparticles in aqueous suspension for coatings applications. Journal of Nanomaterials 2012: 1-10. DOI: https://doi.org/10.1155/2012/718214

Qi J., Ye Y., Wu J., Wang H. \& Li F. (2012). Dispersion and stability of titanium dioxide nanoparticles in aqueous suspension: effects of ultrasonication and concentration. Water Science and Technology 67(1): 147-151.

DOI: https://doi.org/10.2166/wst.2012.545

Staňková M., Jandera P., Škeříková V. \& Urban J. (2013). Cross-linker effects on the separation efficiency on (poly) methacrylate capillary monolithic columns. Part II. Aqueous normal-phase liquid chromatography. Journal of Chromatography A 1289: 47-57.

DOI: https://doi.org/10.1016/j.chroma.2013.03.025

Suslick K.S. (1990). Sonochemistry. Science 247(4949): 1439 1446.

DOI: https://doi.org/10.1126/science.247.4949.1439

Svec F. (2004). Preparation and HPLC applications of rigid macroporous organic polymer monoliths. Journal of Seperation Science 27(10-11): 747-766.

DOI: https://doi.org/10.1002/jssc.200401721

Tang S., Guo Y., Xiong C., Liu S., Liu X. \& Jiang S. (2014). Nanoparticle-based monoliths for chromatographic separations. Analyst 139(17): 4103-4117.

DOI: https://doi.org/10.1039/C4AN00593G

Tong S., Zhou X., Zhou C., Li Y., Li W., Zhou W. \& Jia Q. (2013). A strategy to decorate porous polymer monoliths with graphene oxide and graphene nanosheets. Analyst 138(5): 1549-1557.

DOI: https://doi.org/10.1039/c2an36608h

Wieneke J.U., Kommoß B.R., Gaer O., Prykhodko I. \& Ulbricht M. (2011). Systematic investigation of dispersions 
of unmodified inorganic nanoparticles in organic solvents with focus on the hansen solubility parameters. Industrial and Engineering Chemistry Research 51(1): 327-334.

DOI: https://doi.org/10.1021/ie201973u

Xu Y., Cao Q., Svec F. \& Frechet J.M.J. (2010). Porous polymer monolithic column with surface-bound gold nanoparticles for the capture and separation of cysteine-containing peptides. Analytical Chemistry 82(8): 3352-3358.

DOI: https://doi.org/10.1021/ac1002646

Zhang X., Yin L. \& Pu Y. (2011). Comparison of dispersion of nanoparticles ultrasonicated with probe and cup horn. Journal of Southeast University 27(4): 441-444.
Zhang Y., Wang W., Ma X. \& Jia L. (2016). Polydopamine assisted fabrication of titanium oxide nanoparticles modified column for proteins separation by capillary electrochromatography. Analytical Biochemistry 512: 103-109.

DOI: https://doi.org/10.1016/j.ab.2016.08.015

Zhou S., Wang Y., De Beer T., Baeyens W.R., Fei G.T., Dilinuer M. \& Ouyang J. (2008). Simultaneous separation of eight $\beta$-adrenergic drugs using titanium dioxide nanoparticles as additive in capillary electrophoresis. Electrophoresis 29(11): 2321-2329.

DOI: https://doi.org/10.1002/elps.200700589 